\title{
Quantitative peptidomics of endogenous peptides involved in TGF- $\beta 1$-induced epithelial mesenchymal transition of renal epithelial cells
}

\author{
Rattiyaporn Kanlaya ${ }^{1}$ and Visith Thongboonkerd ${ }^{1}$
}

\begin{abstract}
TGF- $\beta 1$ is a key fibrotic factor mediating epithelial mesenchymal transition (EMT) of epithelial cells through various signaling pathways. However, roles of proteolytic cleavage and endogenous peptide dynamics in TGF- $\beta 1$-induced EMT remain unknown. We therefore performed quantitative peptidomics of TGF- $\beta 1$-induced EMT in renal tubular epithelial cells. The acquired mesenchymal characteristics were confirmed, including morphological change (from cobblestone-like to fibroblast-like), decreased epithelial marker (ZO-1), and increased mesenchymal marker (vimentin). Quantitative peptidomics using stable isotope labeling revealed significantly altered levels of 70 unique endogenous peptides (derived from internal and C-terminal parts of 39 unique precursor proteins) after EMT induction.

Interestingly, the majority of these peptides were derived from non-short-lived proteins, and analysis of P1 position revealed predominance of hydrophobic residues, suggesting that these endogenous peptides were generated mainly from proteasome cleavage. This hypothesis was confirmed by treating the cells with MG132 (a proteasome inhibitor), which provided almost identical endogenous peptide pattern as of the TGF- $\beta 1$-treated cells. Moreover, validation assay showed marked reduction of proteasome peptidase activity in both TGF- $\beta 1$-treated and MG132-treated cells. This is the first peptidome dataset that provides several novel aspects of mechanisms for TGF- $\beta 1$-induced EMT. Our data also suggest that TGF- $\beta 1$ exerts inhibitory effect against proteasome activity during EMT induction.
\end{abstract}

\section{Introduction}

Renal interstitial fibrosis is a common feature of chronic kidney disease (CKD). Patients with CKD are suffered from declined renal function as a consequence of renal injury, nephron loss, and reduction of glomerular filtration rate $\mathrm{e}^{1,2}$. Transforming growth factor- $\beta 1$ (TGF- $\left.\beta 1\right)$ is widely accepted as a major mediator involved in renal fibrosis leading to CKD via various mechanisms, including inflammation, suppression of extracellular matrix (ECM) degradation, activation of residential myofibroblasts, and

Correspondence: Visith Thongboonkerd (thongboonkerd@dr.com) (vthongbo@yahoo.com)

${ }^{1}$ Medical Proteomics Unit, Office for Research and Development, Faculty of Medicine Siriraj Hospital, and Center for Research in Complex Systems Science, Mahidol University, Bangkok, Thailand

Edited by N. Barlev induction of epithelial mesenchymal transition (EMT) that is associated with increased ECM production and accumulation $^{3,4}$. TGF- $\beta 1$ triggers signal transduction through its Type I and Type II receptors by forming a signaling complex that subsequently regulates many downstream molecules (e.g., ERK1/2 and Smads) to further induce fibrotic factors ${ }^{3,5}$. While many signaling pathways mediated by TGF- $\beta 1$ have been characterized, very little is known about roles of TGF- $\beta 1$ and its downstream pathways in regulation of intracellular proteolytic cleavage.

To date, only a few studies have addressed the interplays between cellular protease activities and TGF- $\beta 1$ signaling. The active serine protease, plasmin, has been reported to be involved in TGF- $\beta 1$ activation to promote renal changes were made. The images or other third party material in this article are included in the article's Creative Commons license, unless indicated otherwise in a credit line to the material. If
material is not included in the article's Creative Commons license and your intended use is not permitted by statutory regulation or exceeds the permitted use, you will need to obtain permission directly from the copyright holder. To view a copy of this license, visit http://creativecommons.org/licenses/by/4.0/. 
(A)

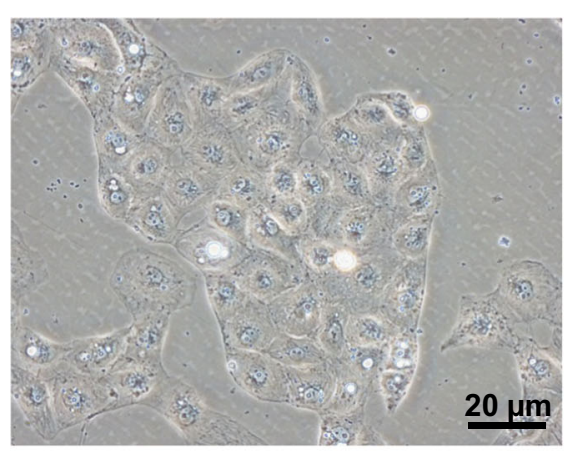

(B)

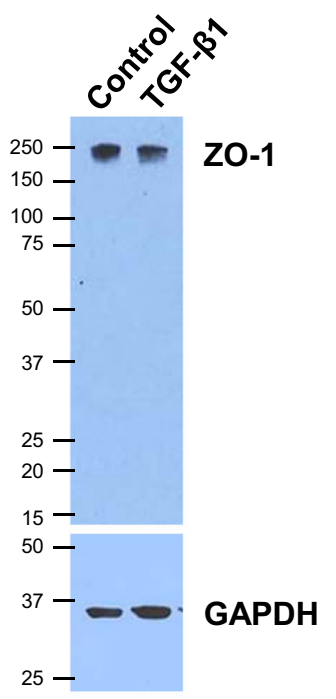

(C)

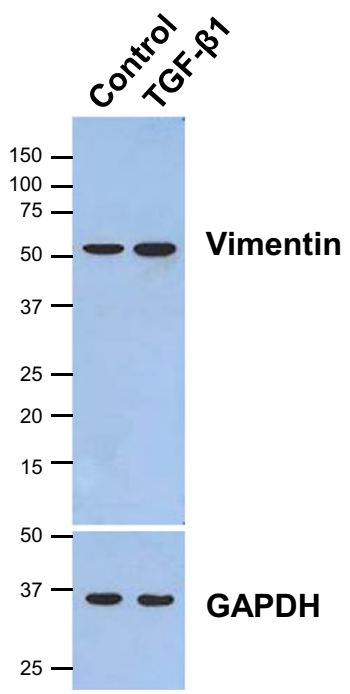

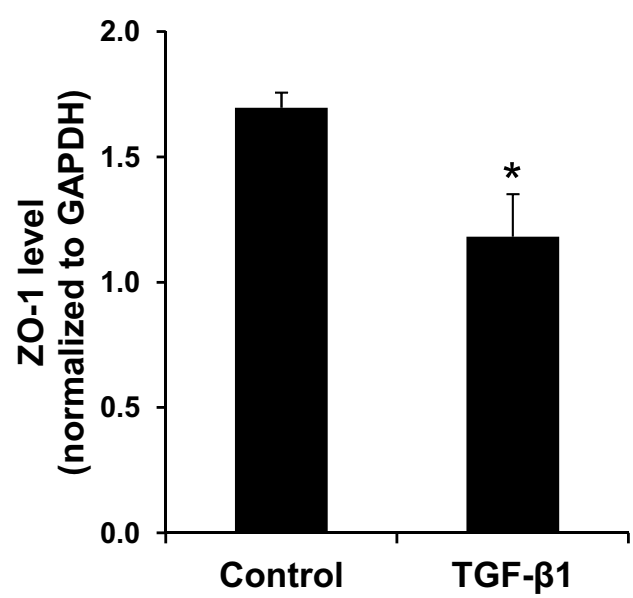

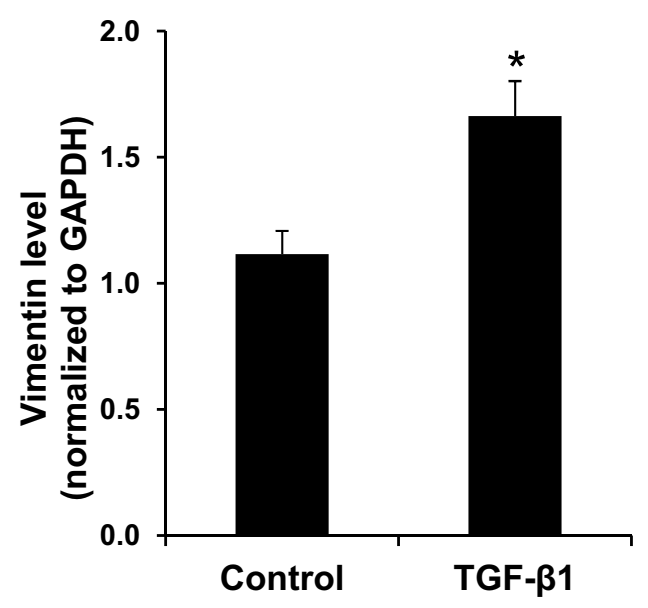

Fig. 1 Induction of EMT in HK-2 cells by TGF- $\boldsymbol{\beta} 1$. a Controlled (untreated) cells had cobblestone like morphology, whereas those treated with 5 $\mathrm{ng} / \mathrm{mL}$ TGF- $\beta 1$ for $48 \mathrm{~h}$ showed fibroblast-like morphology. Original magnification power $=\times 400$. b, c Epithelial marker (ZO-1) was significantly decreased, whereas mesenchymal marker (vimentin) was significantly increased. GAPDH served as the protein loading control. Each bar represents mean \pm SD of the data derived from three independent experiments. ${ }^{*} p<0.05 \mathrm{vs}$. control 


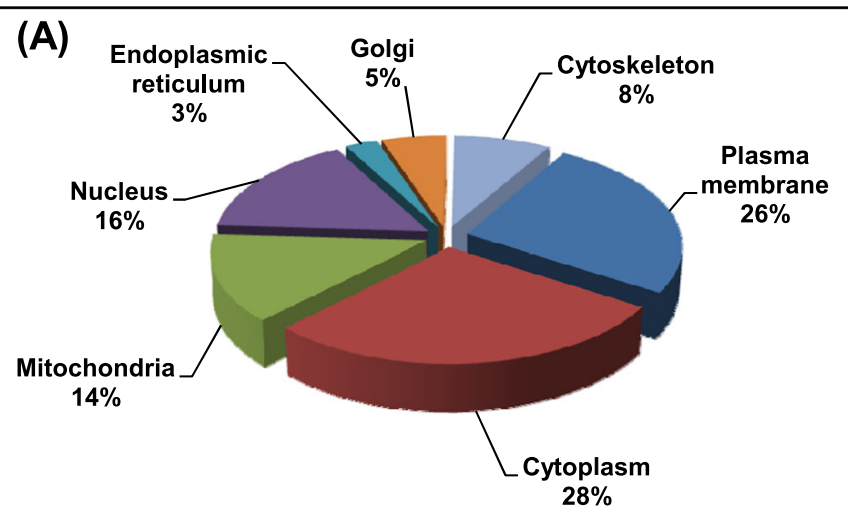

(C)

\section{GO biological process}

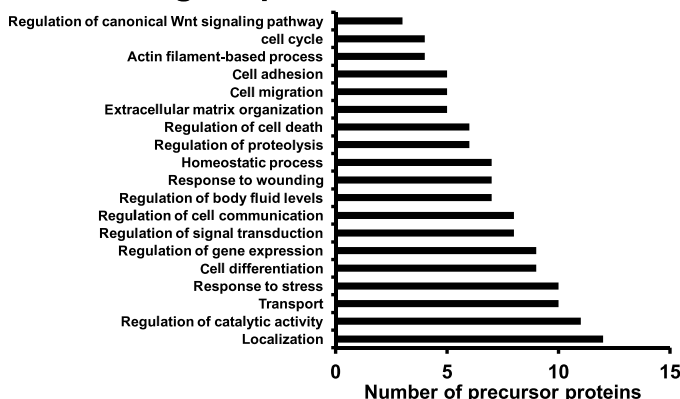

(E)

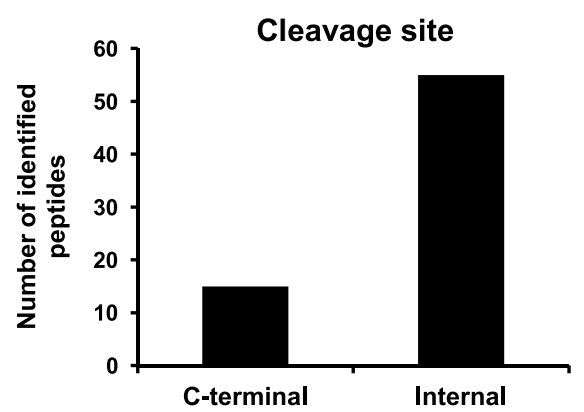

(G)

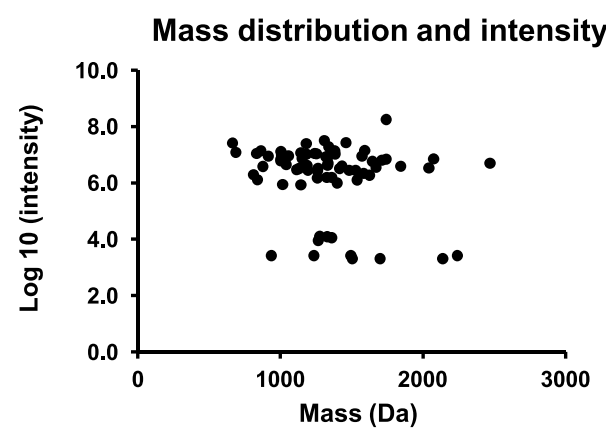

(B)

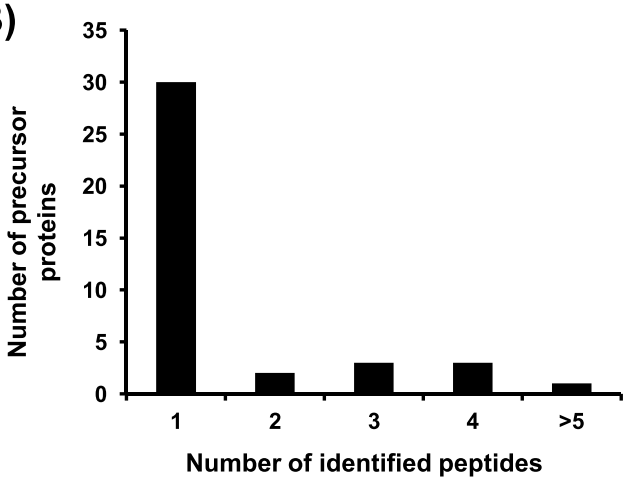

(D)

GO molecular function

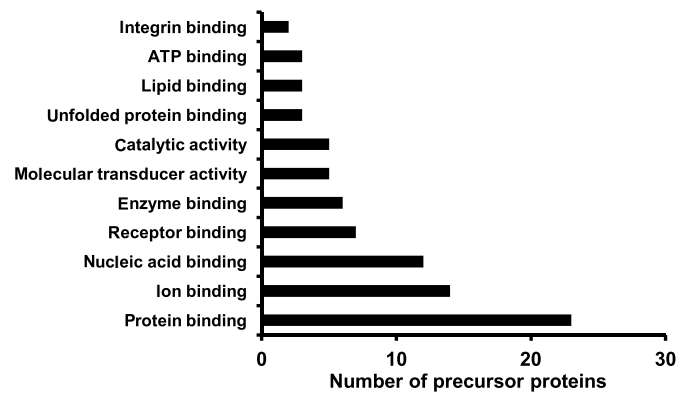

(F)

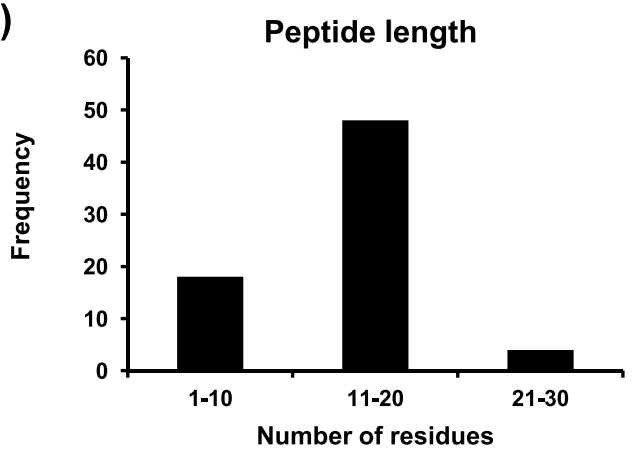

(H)

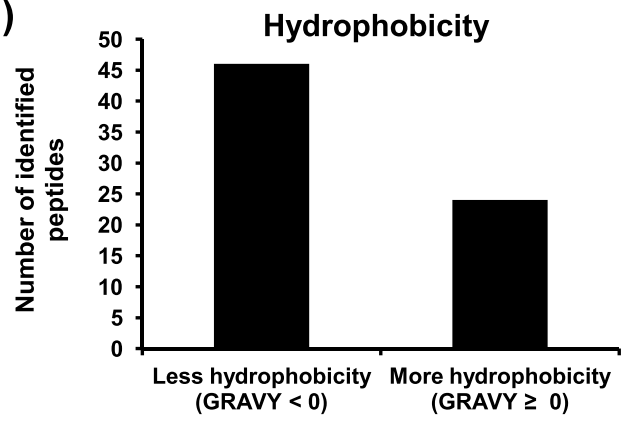

Fig. $\mathbf{2}$ Characteristics of the altered precursor proteins and endogenous peptides in TGF- $\boldsymbol{\beta} 1$-treated cells. The altered precursor proteins were grouped by their subcellular localization (a), number of the identified peptides (b), GO enrichment analysis of biological process (c), and GO molecular function (d). The altered peptides were grouped by their cleavage site (e), length (number of amino acid residues) (f), mass distribution and intensity $(\mathbf{g})$, and hydrophobicity (h) 


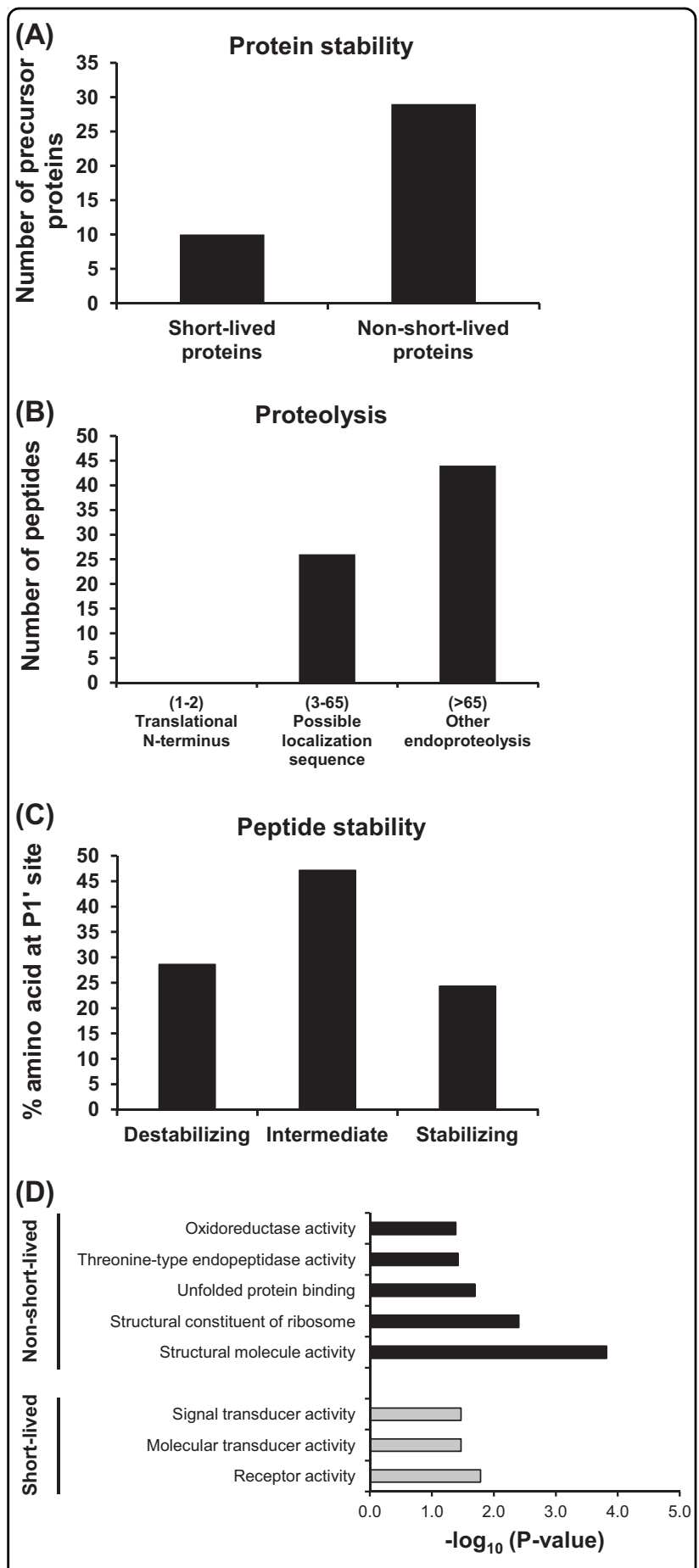

Fig. 3 Stability of the altered precursor proteins and endogenous peptides in TGF- $\beta$ 1-treated cells. a Number of the precursor proteins based on their stability (short-lived vs. non-short-lived proteins). $\mathbf{b}$ Grouping of the endogenous peptides according to their three main categories of proteolysis. $\mathbf{c}$ Peptide stability based on the stability of amino acids at $\mathrm{P} 1^{\prime}$ site. $\mathbf{d}$ GO enrichment analysis of molecular function of the precursor proteins classified by their stability interstitial fibrosis in mice with unilateral ureteral obstruction (UUO), whereas plasmin-deficient mice showed only mild degree of interstitial fibrosis ${ }^{6,7}$. Recent study has demonstrated the potential strategy to inhibit renal fibrosis by using a serine protease inhibitor, camostat mesilate, both in vitro and in vivo ${ }^{8}$. In vitro investigation using a rat kidney fibroblast cell line, NRK-49F, treated with TGF- $\beta 1$ has shown that camostat mesilate could reduce activation of the downstream targets of TGF- $\beta 1$ signaling, i.e., phosphorylated ERK1/2 and $\operatorname{Smad} 2 / 3^{8}$. In vivo study using rat with UUO has also demonstrated that camostat mesilate could suppress TGF- $\beta 1$-mediated renal fibrosis by a marked reduction of collagen accumulation in the kidney ${ }^{8}$. Another study has revealed that a urinary trypsin inhibitor, ulinastatin, could attenuate renal interstitial fibrosis in UUO rats by affecting the TGF- $\beta 1 /$ Smad pathways ${ }^{9}$.

These findings raise a hypothesis that TGF- $\beta 1$ and its downstream molecules may affect protease/peptidase activities to aggravate their pathological roles during the development of renal fibrosis. However, there was no previous study to analyze endogenous peptides generated during TGF- $\beta 1$-induced renal fibrosis or EMT. With the current insights that intracellular peptides bear significant biological functions, the present study thus aimed to characterize the endogenous peptides generated from renal tubular epithelial cells under TGF- $\beta 1$-induced EMT condition by a quantitative peptidomics approach using stable isotopic labeling, followed by bioinformatic prediction and functional validations.

\section{Results}

Induction of EMT by TGF- $\beta 1$ in HK-2 cells

HK-2 cells were treated with $5 \mathrm{ng} / \mathrm{mL}$ TGF- $\beta 1$ for $48 \mathrm{~h}$ and cell morphology was observed under a phase-contrast microscope. The results showed that TGF- $\beta 1$ induced change of the cell morphology to fibroblast-like, whereas the controlled (untreated) cells still had a typical morphology of epithelial cells (Fig. 1a). In addition, western blotting revealed significant decrease in an epithelial marker ZO-1 (Fig. 1b), whereas the mesenchymal marker vimentin was significantly increased in TGF- $\beta 1$-treated cells (Fig. 1c).

\section{Characteristics of the altered endogenous peptides and precursor proteins}

Using stable isotopic labeling, we identified a total of 223 individual endogenous peptides in the TGF- $\beta 1$ treated cells. Quantitative analyses revealed significant changes in levels of 70 unique peptides derived from 39 unique precursor proteins in the TGF- $\beta 1$-treated cells as compared to the controlled cells (Supplementary Table 1). Most of these altered peptides (64 peptides from 34 precursor proteins) were decreased, whereas six peptides 


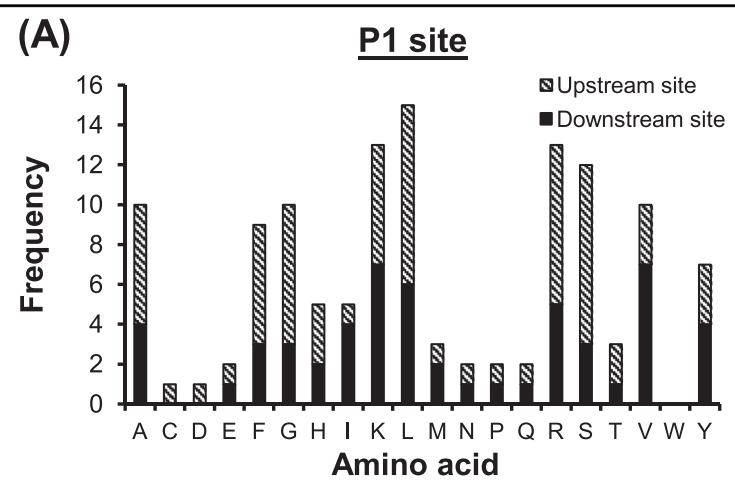

(B)

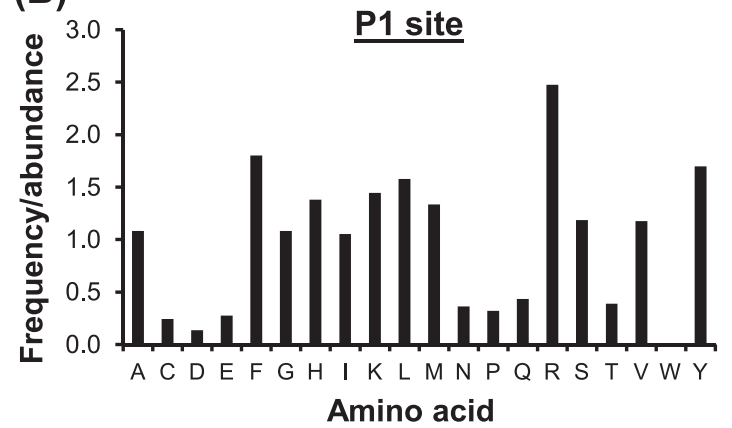

(C)

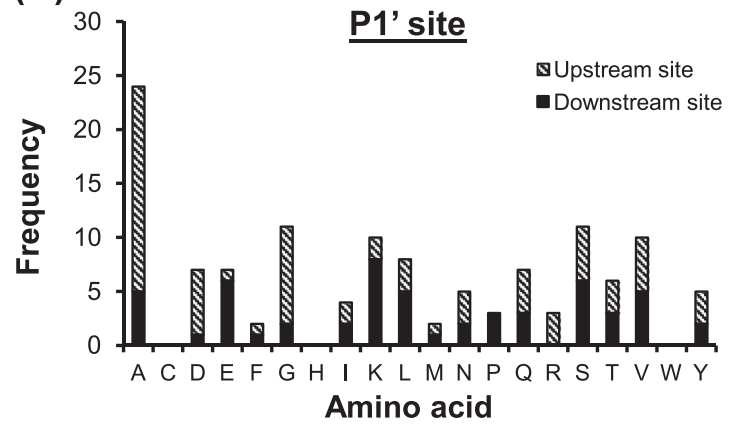

(D)

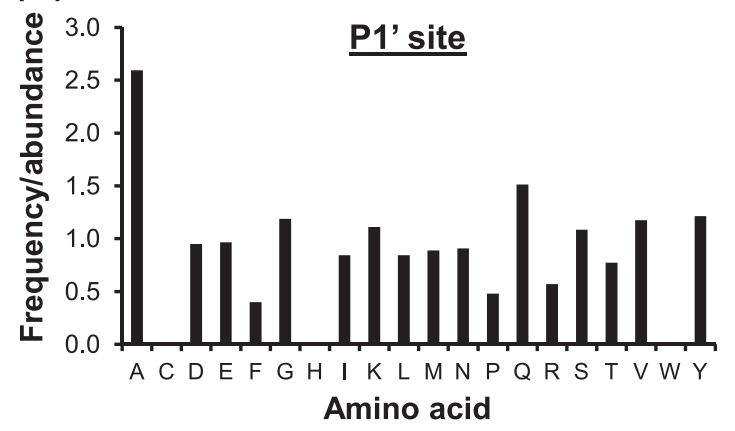

Fig. 4 Cleavage sites of the altered endogenous peptides in TGF$\beta 1$-treated cells. Frequency and frequency normalized with abundance of each amino acid found at P1 site are illustrated in (a) and (b), respectively, whereas those for P1' site are shown in (c) and (d), respectively derived from five precursor proteins were increased in the TGF- $\beta 1$-treated cells. Analysis of the cleavage sites by MEROPs and TopFINDer tools revealed that only $2 / 5$ of the identified altered peptides had known proteolytic enzymes, whereas other $3 / 5$ of them were derived from unknown protease/mechanism (Supplementary Table 1).

Subcellular localization showed that these altered precursor proteins were localized mainly in cytoplasm (28\%), plasma membrane (26\%), nucleus (16\%), and mitochondria (14\%) (Fig. 2a). Frequency of number of the identified peptides from each precursor protein is shown in Fig. 2b. Notably, most of these altered precursor proteins were identified from a single peptide, whereas only one protein was identified with more than five peptides. GO enrichment analysis demonstrated that these altered precursor proteins were involved in many biological processes, e.g., localization, regulation of catalytic activity, transport, response to stress (Fig. 2c). Interestingly, the GO biological processes also included known EMT mechanisms, i.e., cell differentiation, response to wounding, ECM organization, cell migration, actin filament-based process, and regulation of canonical Wnt signaling pathway (Fig. 2c). The enriched GO molecular functions were mainly binding of various cellular molecules, e.g., protein, ion, nucleic acid, receptor, enzyme (Fig. 2d).

The significantly altered endogenous peptides were also characterized for their cleavage sites from the precursor proteins. Most of them were derived from the internal part, whereas some were generated from the C-terminus of the precursor proteins and there were no $\mathrm{N}$-terminusderived peptides observed (Fig. 2e). Interestingly, more than a half of these altered peptides had 11-20 amino acid residues (Fig. 2f). As expected, all of the altered peptides had molecular mass $<3000 \mathrm{Da}$ (most of them were ranged between 1200 and $2000 \mathrm{Da}$ ) (Fig. 2g). Grand average of hydropathicity (GRAVY) was measured by Kyte-Doolittle formula to define hydrophobicity of the peptides ${ }^{10}$. The results showed that the GRAVY indices were mostly $<0$, indicating that most of these peptides were mainly the hydrophilic peptides (Fig. 2h).

\section{Stability of the altered precursor proteins and endogenous peptides}

Protein stability or half-life should be also accounted for the peptide pool. We thus evaluated half-lives of the precursor proteins by using the SProtP tool. The cutoff lifespans of being classified as short-lived and non-shortlived proteins were $<30$ and $>30 \mathrm{~min}$, respectively. The results showed that most of the precursor proteins were non-short-lived proteins (Fig. 3a). Additionally, the altered peptides were grouped into three main categories of proteolytic process (based on the position of proteolytic cleavage on the precursor proteins), including translational $\mathrm{N}$-terminus, localization sequence (signal or transit 
peptide removal), and other endoproteolysis processes. The results showed that most of these peptides were derived from other endoproteolysis processes followed by transit peptide removal (Fig. 3b). Interestingly, no any peptide was derived from translational $\mathrm{N}$-terminus processing.

In addition, the peptide stability was analyzed according to the $\mathrm{N}$-end rule pathway, which is one of the important processes for intracellular protein degradation by proteasome. According to the $\mathrm{N}$-end rule pathway, different amino acids at the $\mathrm{N}$-terminus could affect the protein half-life depending on its stability. Amino acid at the P1' position, which is crucial for the protein half-life, was then analyzed. Interestingly, the intermediate amino acids (S, Y, W, H, A, L, and T with half-life between 1.5 and $20 \mathrm{~h}$ ) and destabilizing amino acids $(\mathrm{Q}, \mathrm{R}, \mathrm{E}, \mathrm{F}, \mathrm{D}, \mathrm{C}, \mathrm{K}$, and $\mathrm{N}$ with half-life $<1.5 \mathrm{~h}$ ) were prominent in these peptides (Fig. 3c). Furthermore, enrichment analysis of molecular function of the precursor proteins based on their half-lives was performed. The statistically significant clusters of molecular functions enriched in non-short-lived proteins included structural molecule activity, structural constituent of ribosome, unfolded protein binding, threoninetype endopeptidase activity, and oxidoreductase activity (Fig. 3d). In contrast, the molecular function enriched in short-lived proteins involved receptor, molecular transducer, and signal transducer activities (Fig. 3d).

Moreover, the endogenous peptides identified in this study were compared to DegraBase, the database of proteolysis in normal and apoptotic human cells ${ }^{11}$. The data showed that only a small number of peptides (nine peptides derived from five different precursor proteins: 10 $\mathrm{kDa}$ heat-shock protein, peptidylprolyl cis-trans isomerase FKBPIA, Peroxiredoxin-5, profilin-1, and vimentin) were overlapped with those deposited in the DegraBase (Supplementary Table 2). All of these peptides were commonly observed in apoptotic cells, whereas some could be found also in healthy cells.

\section{Analysis of the cleavage sites (P1 and P1' positions) of the altered endogenous peptides}

Generally, endogenous peptides require cleavage sites for specific enzyme(s) to recognize and cleave. Internal peptides require both upstream and downstream cleavage sites, whereas $\mathrm{N}$-terminal or $\mathrm{C}$-terminal peptides require only downstream or upstream cleavage site, respectively. According to the general nomenclature of cleavage site positions, $\mathrm{P} 1$ and $\mathrm{P} 1^{\prime}$ are defined as the $\mathrm{N}$-terminal and $\mathrm{C}$ terminal sides of the cleaved peptide bond, respectively ${ }^{12}$. Analysis of the frequency of amino acids at P1 position (both upstream and downstream) revealed that leucine, lysine, arginine, and serine were the most common residues found at P1 position (Fig. 4a). Normalization of the amino acid frequency with abundance of each amino acid in human proteins showed that arginine, phenylalanine, tyrosine, and lysine were most prominent at $\mathrm{P} 1$ position (Fig. 4b). Similar analysis was performed at $\mathrm{P} 1^{\prime}$ position, the results showed that alanine was mostly found (Fig. 4c). Interestingly, after adjusting with amino acid abundance, alanine remained the most prominent residue found at P1' position (Fig. 4d).

\section{Validation of the significant role of proteasome activity to generate endogenous peptides in TGF- $\beta 1$-treated cells}

The proteolytic activity of proteasome depends on its three main subunits, including $\beta 1, \beta 2$, and $\beta 5$, which have caspase-like, trypsin-like, and chymotrypsin-like activities, respectively. These subunits face the inner cylinder of 20S-proteasome structure and altogether cleave proteins into small peptides. To address whether the altered endogenous peptides found in TGF- $\beta 1$-treated cells were derived from proteasome activity, the peptide cleavage sites at P1 position were further analyzed for proteasome cleavage by $\beta 1$-subunit (at acidic residues: $\mathrm{D}$ and $\mathrm{E}$ ), $\beta 2$ subunit (at basic residues: $\mathrm{H}, \mathrm{K}$ and $\mathrm{R}$ ), and $\beta 5$-subunit (at hydrophobic residues: A, F, I, L, V, W, and Y). Other amino acids found at $\mathrm{P} 1$ position $(\mathrm{C}, \mathrm{G}, \mathrm{M}, \mathrm{N}, \mathrm{P}, \mathrm{Q}, \mathrm{S}$, and $\mathrm{T})$ were grouped as non-proteasome cleavage site. For internal peptides that require two cleavage sites, they were grouped into $\beta 1$ - or $\beta 2$-cleaved peptides if either side was found with acidic or basic residue, respectively. The $\beta 5$ cleaved peptides were considered when both sides were found with hydrophobic residues. The results revealed that $64 \%$ of the altered endogenous peptides contained the proteasome cleavage sites (Fig. $5 \mathrm{a}$ ).

To validate such prediction, an additional experiment was performed. Endogenous peptides were extracted from the cells treated with MG132, the proteasome inhibitor, and then analyzed for the amino acid residue at P1 position. The data showed that MG132 caused significant changes in levels of 210 unique peptides derived from 75 unique proteins in MG132-treated cells (Supplementary Table 3). As expected, $74 \%$ of the endogenous peptides were derived from proteasome cleavage (Fig. 5b), consistent with the data observed from initial prediction in the TGF- $\beta 1$-treated cells (Fig. 5a). To further address whether TGF- $\beta 1$ could inhibit proteasome activity, only the altered peptides that were identified from both TGF$\beta 1$ - and MG132-treated cells were analyzed. The data showed that levels of all these common altered peptides were decreased when compared to the controlled (untreated) cells and their cleavage sites were attributed mainly from the activity of $\beta 5$-subunit of proteasome (Fig. 5c). Interestingly, patterns of these altered peptides induced by the two different treatments were almost identical, confirming that TGF- $\beta 1$ exerted inhibitory effect against proteasome activity during EMT development in renal tubular epithelial cells. 
Finally, direct measurement of proteasome peptidase activity was performed to further confirm our hypothesis. The data showed that proteasome peptidase activity was marked reduced (by approximately a half) in both TGF- $\beta 1$-treated and MG132-treated cells as compared to the controlled cells (Fig. 6). And such activity was comparable (almost equal) in TGF- $\beta 1$-treated and MG132-treated conditions (Fig. 6).

\section{Discussion}

Peptidomics is a comprehensive study of peptide contents (termed as "peptidome") in a biological sample that may be naturally derived from small coding RNA or intracellular proteolytic cleavage ${ }^{13,14}$. Initially, peptidomics analyses had been conducted mainly for studying peptide hormones or neuropeptides ${ }^{15,16}$. Indeed, naturally occurred endogenous peptides can be found in other tissues and cells ${ }^{17,18}$. Intracellular peptides are postulated to play crucial roles in a vast variety of cellular functions, including regulation of protein-protein interactions ${ }^{19}$ and intracellular signal transduction ${ }^{20}$. Importantly, several studies have revealed clinical impact of intracellular peptides in biomarker discovery for many kidney diseases, e.g., acute kidney injury ${ }^{21}$, acute renal allograft rejection ${ }^{22}$, diabetic nephropathy ${ }^{23,24}$, and CKD ${ }^{25}$.

In the present study, we performed quantitative peptidomics analysis of TGF- $\beta 1$-induced EMT in renal tubular epithelial cells. When the cells underwent EMT, a set of endogenous peptides were altered in their levels as compared to the controlled or untreated cells. Interestingly, most of the peptides had decreased levels and were derived from non-shorted-lived proteins. Additionally, only a small number of these peptides were generated from known enzymatic cleavage, while most of them were derived from unknown protease/mechanism. However, after analysis of the peptide cleavage sites, the results suggested that both known proteolytic enzymes (e.g., matriptase-2, maprin, and matrix metallopeptidase-2) and unknown proteases found in this study were most likely the members of serine protease, which is the major protease family responsible for protein degradation ${ }^{26}$. In addition to the known biological processes related to EMT, the clusters of precursor proteins involving regulation of catalytic activity and transport were significantly enriched. In concordance with our results, Cha et $\mathrm{al}^{27}$ have previously highlighted the importance of metabolic trade-off between anabolic and catabolic states of cancer cells during EMT in order to survive under metabolic stress. Moreover, recent findings in experimental animals with fibrosis and cultured cells have shown the deregulation of several transporters in fibrotic kidney and TGF- $\beta 1$-treated HK- 2 cells $^{28}$. These findings support that diverse cellular functions, including catabolic process and cellular transport, are deregulated during EMT.

Previous study has revealed the role of proteolytic processing by lysosomal pathway in cancer cell invasion ${ }^{29}$.
The data have shown that lysosomal proteins (in particular, cysteine cathepsins) were increased in TGF- $\beta 1$ induced EMT in mammary cancer cells and facilitated cell invasion. Interestingly, inhibition of cathepsin activity could reduce cell invasion ${ }^{29}$. It is known that proteolytic cleavage by lysosomal pathway is crucial for maintaining cellular homeostasis. In the present study, we also identified the altered peptides that were derived from cathepsin C (also known as dipeptidyl peptidase 1) and cathepsin D. These findings strengthened the important role of lysosomal proteolytic cleavage for regulation of the cellular homeostasis.

Characterizations of the altered peptides induced by TGF- $\beta 1$ in our present study also revealed that their sizes were ranged from 6 to 30 residues, while the masses were $<3000 \mathrm{Da}$ for all the peptides identified. Additionally, proportion of these peptides showed that the peptides with larger size were less abundant. Moreover, analysis of the cleavage site at P1 position showed that the hydrophobic residues were more prominent. These characteristics of the peptides were most likely due to proteasome activity $^{30}$. To decipher the contribution of proteasome to peptide generation in TGF- $\beta 1$-treated cells, we thus used a proteasome inhibitor (MG132) to inhibit the proteasome activity. MG132 is a peptide aldehyde (carbobenzylLeu-Leu-Leu-aldehyde) that can strongly inhibit $\beta 1$ (caspase-like) and $\beta 5$ (chymotrypsin-like) subunits of $20 \mathrm{~S}$ proteasome $^{31}$. The concentration of MG132 used in this study was based on that applied to a previous study demonstrating that $0.5 \mu \mathrm{M}$ was the lowest concentration of MG132 to inhibit proteasome activity without any effect on viability of $\mathrm{HK}-2$ cells $^{32}$. As expected, the majority of the altered endogenous peptides in the MG132-treated HK-2 cells were decreased, confirming that proteasome played significant role in proteolytic cleavage to generate endogenous peptides in HK-2 renal tubular cells.

The catalytic activity of proteasome, in particular $\beta 2$ and $\beta 5$ subunits, has been demonstrated to be suppressed in TGF- $\beta 1$-induced EMT in breast mammary cancer cells ${ }^{29}$. Additionally, treatment with specific inhibitors of these two subunits was associated with the increase in EMTrelated genes/proteins ${ }^{33}$. In concordance, our present study also showed that most of the endogenous peptides extracted from TGF- $\beta 1$-treated cells were decreased in their levels. The results were consistent when the cells were treated with the proteasome inhibitor MG132. Moreover, levels of all the altered peptides commonly found in both TGF- $\beta 1$ - and MG132-treated cells were decreased. Comparing to the quantitative studies of intracellular peptidome, our data were consistent with the previous findings demonstrating that non-borate inhibitors (including epoxomicin, clasto-lactacystin $\beta$-lactone, and MG132) caused markedly decreases of the 


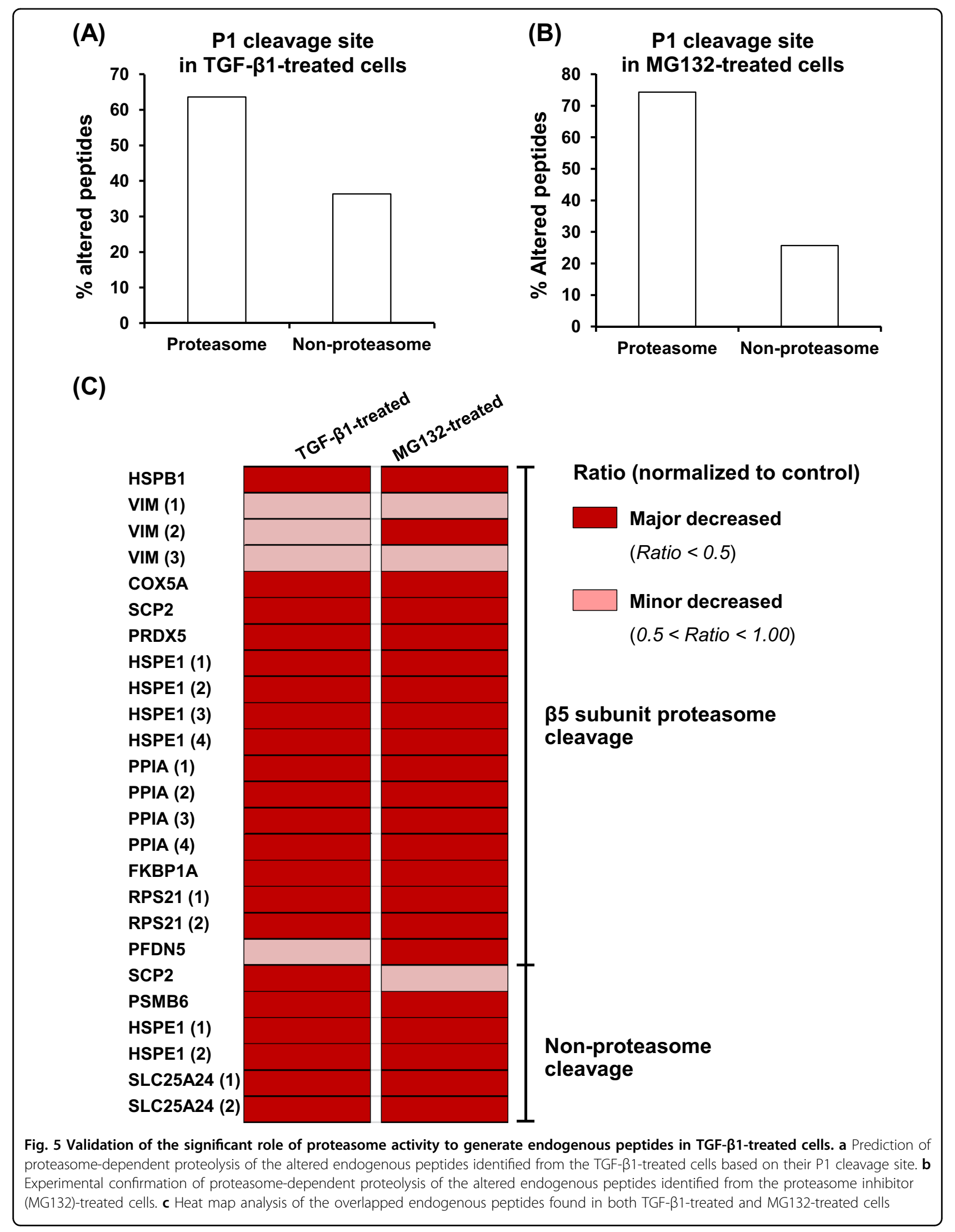




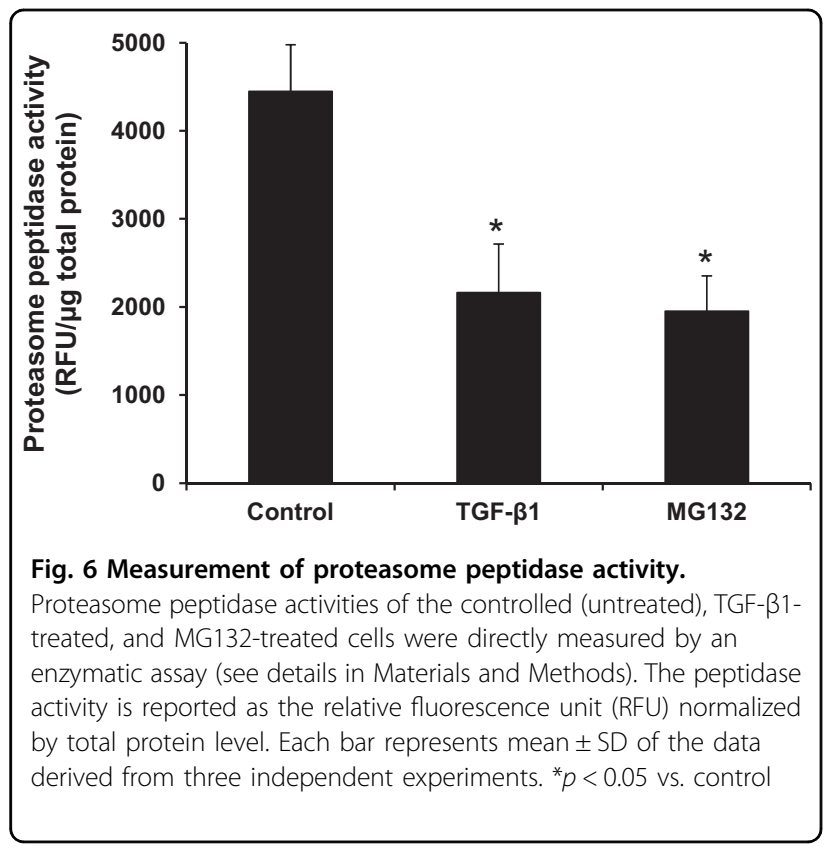

intracellular peptides ${ }^{32,34}$. Additionally, we found several peptides derived from 40 S ribosomal protein S21, cytochrome $C$ oxidase subunit 5 , peptidylprolyl isomerase A, and peroxiredoxin -5 had significantly decreased levels in both TGF- $\beta 1$ - and MG132-treated cells, consistent with those previously reported in the cells treated with various proteasome inhibitors ${ }^{32,34}$. Collectively, our findings thus confirmed the hypothesis that TGF- $\beta 1$ exerted inhibitory effect against proteasome activity, resulting in the decreased levels of endogenous peptides produced in TGF- $\beta 1$-treated cells. One might argue that the treatment itself would cause accumulation of precursor proteins, thereby subsequently reduced intracellular peptide pool. However, previous systematic evaluation of protein degradation by ubiquitin-modified proteome using quantitative proteomics approach has revealed that most of the cellular proteins were not accumulated when the proteasome activity was inhibited ${ }^{35,36}$. Moreover, direct measurement of proteasome peptidase activity in the present study clearly demonstrated the markedly reduced peptidase activity of proteasome in TGF- $\beta 1$-treated cells as compared to the controlled cells, consistent with the data observed in the MG132-treated condition. Taken together, the decreased peptide levels observed in our present study was most likely resulted from the inhibition of proteasome activity, indicating that the majority of endogenous peptides generated in TGF- $\beta 1$-treated HK- 2 were derived from proteasome cleavage.

In conclusion, we demonstrate herein alterations in peptidome of renal tubular epithelial cells after induction of EMT by TGF- $\beta 1$. Our data also revealed the role of proteasome in generation of endogenous peptides during the physiologic condition. Moreover, TGF- $\beta 1$ exerted inhibitory effect on proteasome activity that might be another relevant mechanism of TGF- $\beta 1$ to induce EMT. The information regarding regulation of proteolytic cleavage can be added to a growing list of molecular mechanisms of renal fibrosis mediated by TGF- $\beta 1$ and may facilitate the ultimate goal to define effective strategy to prevent CKD.

\section{Materials and methods}

\section{Cell culture and treatment}

A human proximal renal tubular cell line (HK-2) was cultivated in a growth medium containing Dulbecco's modified Eagle's medium (DMEM) (Gibco, Grand Island, NY) supplemented with $10 \%$ inactivated fetal bovine serum (FBS) (Gibco) in the presence of $100 \mathrm{U} / \mathrm{mL}$ penicillin $\mathrm{G}$ and $100 \mathrm{mg} / \mathrm{mL}$ streptomycin (Sigma; St. Louis, $\mathrm{MO})$. The cells were maintained in a humidified incubator at $37^{\circ} \mathrm{C}$ with $5 \% \mathrm{CO}_{2}$.

To induce EMT, HK-2 cells were seeded at a density of $5 \times 10^{4}$ cells/well in six-well plate and cultured in the growth medium for $24 \mathrm{~h}$. The growth medium was then replaced with serum-free DMEM for $6 \mathrm{~h}$ followed by treatment with $5 \mathrm{ng} / \mathrm{mL}$ TGF- $\beta 1$ (BioLegend, San Diego, $\mathrm{CA}$ ) in a maintenance medium containing DMEM supplemented with $1 \%$ FBS for further $48 \mathrm{~h}$. The cells without TGF- $\beta 1$ treatment served as the control. Changes in cell morphology were observed under a phase-contrast microscope (Olympus CKX41; Olympus Co. Ltd, Tokyo, Japan). The cells were then subjected to endogenous peptide extraction and quantitative peptidomics analysis.

\section{Western blotting}

At the indicated time-point, the cells were harvested and lyzed by Leammli's buffer. Protein concentration was quantified by Bradford's method using Bio-Rad Protein Assay (Bio-rad, Milano, Italy). Proteins with an equal amount of $30 \mu \mathrm{g}$ per each sample were resolved by $12 \%$ SDS-PAGE. The resolved protein bands were electrophoretically transferred onto a nitrocellulose membrane and non-specific bindings were blocked by $5 \%$ skim milk in phosphate-buffered saline (PBS). The membrane was then probed with rabbit polyclonal anti-ZO-1 (Santa Cruz Biotechnology, Santa Cruz, CA) or mouse monoclonal anti-vimentin (Santa Cruz Biotechnology) antibody (both at a dilution of $1: 1000$ in $1 \%$ skim milk in PBS) at $4{ }^{\circ} \mathrm{C}$ overnight. After washing with PBS, the membrane was incubated with horseradish peroxidase-conjugated swineanti-rabbit IgG or rabbit-anti-mouse IgG secondary antibody (Dako, Denmark A/S, Denmark) (both at a dilution of $1: 2000$ in $1 \%$ skim milk/PBS). Detection of GAPDH was performed to serve as an equal loading control using mouse monoclonal anti-GAPDH (Santa Cruz Biotechnology) (1:1000 in 1\% skim milk/PBS) as a 
primary antibody. The reactive protein bands were visualized by SuperSignal West Pico chemiluminescence substrate (Pierce Biotechnology, Inc., Rockford, IL) and autoradiography. Band intensity was quantitated using ImageMaster 2D Platinum software (GE Healthcare, Uppsala, Sweden).

\section{Extraction of endogenous peptides and measurement of peptide concentration}

The cells were harvested by trypsinization from three independent $100-\mathrm{mm}$ culture dishes per each group. The endogenous peptides were extracted as previously described $^{37}$. Briefly, the cell pellet was collected by centrifugation at $800 \mathrm{~g}$ for $5 \mathrm{~min}$ at $4{ }^{\circ} \mathrm{C}$. Thereafter, the pellet was added with $1 \mathrm{~mL}$ hot water $\left(80^{\circ} \mathrm{C}\right)$ and further incubated at $80^{\circ} \mathrm{C}$ for $20 \mathrm{~min}$. The sample was centrifuged at $13,000 \mathrm{~g}$ and $4{ }^{\circ} \mathrm{C}$ for $5 \mathrm{~min}$ and then kept at $-80^{\circ} \mathrm{C}$ overnight. After thawing at room temperature (set at $25^{\circ}$ C), the sample was centrifuged at $13,000 \mathrm{~g}$ and $4{ }^{\circ} \mathrm{C}$ for 15 min. The supernatant was collected and sample volume was reduced by a half using a vacuum concentrator (Savant, Holbrook, NY). The remaining supernatant was acidified with ice-cold $0.1 \mathrm{M} \mathrm{HCl}$ for $15 \mathrm{~min}$ on ice (to make a final concentration of $10 \mathrm{mM} \mathrm{HCl}$ ). After centrifugation at $13,000 \mathrm{~g}$ and $4{ }^{\circ} \mathrm{C}$ for $40 \mathrm{~min}$, the supernatant containing crude endogenous peptides was further isolated by a Nanosep 10K device (Pall Corporation, Port Washington, NY). The flow-through fraction was collected and dried by using a vacuum concentrator. Peptide concentration was determined by BCA assay (Pierce Biotechnology, Inc.) as described previously ${ }^{38}$.

\section{Labeling the endogenous peptides with stable isotope}

Dimethyl stable isotope labeling was performed using C18 stage tip packed with POROS R3 beads (Applied Biosystems, Waltham, MA). Initially, the $\mathrm{C} 18$ stage tips were washed twice with $100 \mu \mathrm{L}$ acetonitrile (ACN) and equilibrated twice with $100 \mu \mathrm{L} 1 \%$ trifluoroacetic acid (TFA). Endogenous peptides were dissolved in $100 \mu \mathrm{L}$ of $1 \%$ TFA and applied to the stage tips, followed by washing with $100 \mu \mathrm{L}$ of $0.1 \%$ TFA twice. The light and heavy labeling solutions were prepared separately. The labeling reagent was prepared in $50 \mathrm{mM}$ sodium phosphate buffer (pH 7.5) containing $50 \mu \mathrm{L}$ of $4 \%(\mathrm{v} / \mathrm{v})$ of either $\mathrm{CH}_{2} \mathrm{O}$ (light) (Sigma) or $\mathrm{CD}_{2} \mathrm{O}$ (heavy) (Cambridge Isotope Laboratories, Tewksbury, MA) and $250 \mu \mathrm{L}$ of $0.6 \mathrm{M}$ sodium cyanoborohydride $\left(\mathrm{NaBH}_{3} \mathrm{CN}\right)$ (Sigma). The stage tips were applied five times with $100 \mu \mathrm{L}$ labeling reagent separately (allowing the reaction for $5 \mathrm{~min}$ each time). The peptides extracted from the controlled and TGF- $\beta 1$ treated cells were separately incubated with the light and heavy labeling solutions, respectively. After labeling, the stage tips were washed twice with $100 \mu \mathrm{L}$ of $0.1 \%$ TFA and the peptides were eluted with $100 \mu \mathrm{L}$ of $50 \% \mathrm{ACN}$ in $0.1 \%$
TFA followed by $100 \mu \mathrm{L}$ of $70 \%$ ACN in $0.1 \%$ TFA. An equal amount of the labeled peptides from each condition was mixed and dried using vacuum concentrator. The peptides were finally dissolved in $0.1 \%$ formic acid (FA) and further analyzed by nanoLC-MS/MS.

\section{NanoLC-MS/MS using LTQ Orbitrap-XL mass spectrometer and data analyses}

The peptide mixture was resuspended in $5 \mu \mathrm{L}$ of $0.1 \%$ FA and separated by nanoflow liquid chromatography (nanoLC) using the EASY-nLC system (Thermo Scientific, Waltham, MA) equipped with a pre-column (EASYColumn, 2-cm-long, 100- $\mu \mathrm{m}$-diameter) packed with 5 - $\mu \mathrm{m}$ C18-A1 (Thermo Scientific) connected to an analytical column (EASY-Column, 10-cm-long, 75- $\mu \mathrm{m}$-diameter) packed with 3- $\mu \mathrm{m}$ C18-A2 (Thermo Scientific). Twomobile-phase system consisting of buffer A ( $0.1 \%$ FA) and buffer B (95\% ACN/0.1\% FA) was used. The peptides were separated using a linear gradient of $0-45 \%$ buffer B over $160 \mathrm{~min}$ at a flow rate of $200 \mathrm{~nL} / \mathrm{min}$. The eluted peptides were directly electrosprayed into the LTQ Orbitrap-XL mass spectrometer (Thermo Scientific), which was operated in a collision-induced dissociation top-12 mode under the control of the Xcalibur 2.1.0 and LTQ Tune Plus 2.5.5 software (Thermo Scientific). The cycle of one full scan was performed at a resolution of 30,000 $(350-1800 \mathrm{~m} / \mathrm{z}$ mass range) followed by 12 datadependent MS/MS scans at a resolution of 7500 . The normalized collision energy at $47 \%$ and the activation time at $10 \mathrm{~ms}$ were set for acquiring mass spectra. Labeled peptide samples prepared from three biological replicates per each group were subjected to nanoLC-MS/MS analyses in technical triplicate (a total of nine MS/MS runs were performed for each group).

The resulting files were analyzed by Proteome Discoverer v.1.4 (Thermo Scientific). The workflow included peptide identification and quantification. The SEQUEST algorithm was used to search against Human Swiss-Prot FASTA database (with 20,205 entries as of June 2017). The search parameters included proteolytic enzyme $=$ none; precursor mass tolerance $=10 \mathrm{ppm}$; and fragment mass tolerance $=0.6 \mathrm{Da}$. Oxidation of methionine, dimethyl (K), dimethyl (N-term), dimethyl:2H(4) (K), and dimethyl:2H(4) (N-term) were selected as dynamic modifications. The target false discovery rate was set at $<1 \%$ by performing a decoy database search. SEQUEST parameters were configured as follows: delta $\mathrm{cn} \geq 0.05$, Xcorr $\geq 2.0$ for $2+, \geq 2.5$ for $3+$, and $\geq 3.0$ for $>3+$ peptide. Precursor ions quantifier processing mode embedded in the Proteome Discoverer was used for quantifying peptides and ratio of the peak intensity of heavy (treated sample) vs. light (controlled sample) labeled corresponding peptide was obtained. Peptides that were detected only in the control are reported as ratio $=0.01$, whereas 
those detected only in the treated sample are reported as ratio $=100$.

\section{Bioinformatics analyses}

Annotation of proteolytic cleavage and other relevant information of the identified peptides were acquired using various peptidase/peptidome databases, including MEROPS (http://merops.sanger.ac.uk), TopFINDer (http:// clipserve.clip.ubc.ca/topfind/topfinder), DegraBase (https://wellslab.ucsf.edu/degrabase), and SProtP (http:// reprod.njmu.edu.cn/sprotp) ${ }^{11,39-41}$. STRING tool (http:// string-db.org) was used to classify protein precursors according to their biological processes and molecular functions ${ }^{42}$. ProteinCenter software (Thermo Scientific) was used for classification of subcellular localization of the precursor proteins. Grand average of hydropathicity (GRAVY) index computed by using ProtParam tool (http://web.expasy.org/protparam) was used to determine hydrophobicity of the peptides ${ }^{10}$.

\section{Proteasome inhibition}

To inhibit proteasome activity, the cell monolayer was treated with $0.5 \mu \mathrm{M}$ MG132 (a proteasome inhibitor) (Tocris Bioscience, Bristol, UK) in the maintenance medium for $8 \mathrm{~h}$ prior to endogenous peptide extraction and quantitative peptidomics and bioinformatics analyses as aforementioned.

\section{Measurement of proteasome peptidase activity}

Proteasome peptidase activity was measured using the protocol previously described by Valera et al. ${ }^{43}$ with slight modification. Briefly, after treatment with TGF- $\beta 1$ or MG132 as aforementioned, the monolayers of controlled (untreated), TGF- $\beta 1$-treated, and MG132-treated cells were washed with PBS three times, harvested by gentle scrapping, and disrupted with a lysis buffer containing $50 \mathrm{mM}$ HEPES (4-(2-hydroxyethyl)-1-piperazineethanesulfonic acid) (pH 7.8), $10 \mathrm{mM} \mathrm{NaCl}, 1.5 \mathrm{mM}$ $\mathrm{MgCl}_{2}, 1 \mathrm{mM}$ EDTA (ethylenediaminetetraacetic acid), 1 mM EGTA (ethylenebis(oxyethylenenitrilo)tetraacetic acid), $250 \mathrm{mM}$ sucrose, and $5 \mathrm{mM}$ DTT prior to two cycles of 5-s pulse-on and 5-s pulse-off sonication with $70 \%$ amplitude on ice using Sonics Vibra-Cell VCX130 (Sonic \& Materials Inc., Newtown, CT, USA). After centrifugation at $16,000 \mathrm{~g}$ for $10 \mathrm{~min}$ at $4{ }^{\circ} \mathrm{C}, 50 \mu \mathrm{L}$ of the collected supernatant was added to $200 \mu \mathrm{L}$ of the assay buffer (containing $2 \mathrm{mM}$ ATP, $50 \mathrm{mM}$ HEPES (pH 7.8), $10 \mathrm{mM} \mathrm{NaCl}, 1.5 \mathrm{mM} \mathrm{MgCl}_{2}, 1 \mathrm{mM}$ EDTA, 1 mM EGTA, $250 \mathrm{mM}$ sucrose, and $5 \mathrm{mM}$ DTT). Subsequently, a specific substrate ( $N$-succinyl-Leu-Leu-Val-Tyr-amino-4methylcoumarin) (Bachem AG, Bubendorf, Switzerland) was added to the mixture to make its final concentration at $100 \mu \mathrm{M}$. The enzymatic reaction was allowed at $37^{\circ} \mathrm{C}$ for $25 \mathrm{~min}$ and the released fluorescence signals were measured at $\lambda 360 / 460 \mathrm{~nm}$ (excitation/emission wavelength) using a multi-mode microplate reader (BioTek Instruments, Inc., Winooski, VT). The proteasome peptidase activity was finally reported as relative fluorescence unit (RFU) normalized by total protein level as determined by the Bio-Rad Protein Assay (Bio-Rad, Milano, Italy).

\section{Statistical analysis}

Quantitative data are reported as mean $\pm \mathrm{SD}$, unless stated otherwise. Mean difference between two groups was analyzed by Student's $T$-test, whereas multiple comparisons were performed by one-way analysis of variance (ANOVA) with Tukey's post-hoc test using SPSS (version 11.5). Statistical significance was considered at a $p$-value less than 0.05 .

\section{Acknowledgements}

We thank Ms. Angkhana Nilnumkhum for her technical assistance. This study was supported by the Thailand Research Fund (TRG5780294, RTA5680004, IRG5980006, and IRN60W0004), Mahidol University research grant, and Office of the Higher Education Commission and Mahidol University under the National Research Universities Initiative. V.T. and R.K. are also supported by the "Chalermphrakiat" Grant, Faculty of Medicine Siriraj Hospital.

\section{Author contributions}

RK and VT designed research, analyzed data, and wrote the manuscript; R.K performed experiments; and all authors reviewed the manuscript.

\section{Competing interests}

The authors declare that they have no competing interests.

\section{Publisher's note}

Springer Nature remains neutral with regard to jurisdictional claims in published maps and institutional affiliations.

Supplementary Information accompanies this paper at https://doi.org/ 10.1038/s41420-017-0001-x.

Received: 10 August 2017 Revised: 19 September 2017 Accepted: 29 September 2017

Published online: 06 February 2018

\section{References}

1. Lopez-Novoa, J. M., Rodriguez-Pena, A. B., Ortiz, A., Martinez-Salgado, C. \& Lopez Hernandez, F. J. Etiopathology of chronic tubular, glomerular and renovascular nephropathies: clinical implications. J. Transl. Med. 9, 13 (2011).

2. Yamaguchi, J., Tanaka, T. \& Nangaku, M. Recent advances in understanding of chronic kidney disease. F1000Res 4, pii:F1000 (2015).

3. Lopez-Hernandez, F. J. \& Lopez-Novoa, J. M. Role of TGF-beta in chronic kidney disease: an integration of tubular, glomerular and vascular effects. Cell Tissue Res. 347, 141-154 (2012).

4. Gewin, L. \& Zent, R. How does TGF-beta mediate tubulointerstitial fibrosis? Semin. Nephrol. 32, 228-235 (2012).

5. Lan, H. Y. Diverse roles of TGF-beta/Smads in renal fibrosis and inflammation. Int. J. Biol. Sci. 7, 1056-1067 (2011).

6. Munger, J. S. et al. Latent transforming growth factor-beta: structural features and mechanisms of activation. Kidney Int. 51, 1376-1382 (1997).

7. Edgtton, K. L., Gow, R. M., Kelly, D. J., Carmeliet, P. \& Kitching, A. R. Plasmin is not protective in experimental renal interstitial fibrosis. Kidney Int. 66, 68-76 (2004). 
8. Morinaga, J. et al. The antifibrotic effect of a serine protease inhibitor in the kidney. Am. J. Physiol. Renal Physiol. 305, F173-F181 (2013).

9. Ning, X. H., Ge, X. F., Cui, Y. \& An, H. X. Ulinastatin inhibits unilateral ureteral obstruction-induced renal interstitial fibrosis in rats via transforming growth factor beta (TGF-beta)/Smad signalling pathways. Int. Immunopharmacol. 15, 406-413 (2013).

10. Kyte, J. \& Doolittle, R. F. A simple method for displaying the hydropathic character of a protein. J. Mol. Biol. 157, 105-132 (1982).

11. Crawford, E. D. et al. The DegraBase: a database of proteolysis in healthy and apoptotic human cells. Mol. Cell. Proteom. 12, 813-824 (2013).

12. Schechter, I. \& Berger, A. On the size of the active site in proteases. I. Papain. Biochem. Biophys. Res. Commun. 27, 157-162 (1967).

13. Ferro, E. S., Rioli, V., Castro, L. M. \& Fricker, L. D. Intracellular peptides: from discovery to function Emer. EuPA Open Proteom. 3, 143-151 (2014).

14. Dallas, D. C. et al. Current peptidomics: applications, purification, identification, quantification, and functional analysis. Proteom. 15, 1026-1038 (2015).

15. Schrader, M., Schulz-Knappe, P. \& Fricker, L. D. Historical perspective of peptidomics. EuPA Open Proteom. 3, 171-182 (2014).

16. Romanova, E. V. \& Sweedler, J. V. Peptidomics for the discovery and characterization of neuropeptides and hormones. Trends Pharmacol. Sci. 36 579-586 (2015).

17. Gelman, J. S., Sironi, J., Castro, L. M., Ferro, E. S. \& Fricker, L. D. Hemopressins and other hemoglobin-derived peptides in mouse brain: comparison between brain, blood, and heart peptidome and regulation in Cpefat/fat mice. J. Neurochem. 113, 871-880 (2010).

18. Gelman, J. S., Sironi, J., Castro, L. M., Ferro, E. S. \& Fricker, L. D. Peptidomic analysis of human cell lines. J. Proteome Res. 10, 1583-1592 (2011).

19. Fricker, L. D. Analysis of mouse brain peptides using mass spectrometrybased peptidomics: implications for novel functions ranging from nonclassical neuropeptides to microproteins. Mol. Biosyst. 6, 1355-1365 (2010).

20. Haynes, C. M., Yang, Y., Blais, S. P., Neubert, T. A. \& Ron, D. The matrix peptide exporter HAF-1 signals a mitochondrial UPR by activating the transcription factor ZC376.7 in C. elegans. Mol. Cell 37, 529-540 (2010).

21. Metzger, J. et al. Urinary excretion of twenty peptides forms an early and accurate diagnostic pattern of acute kidney injury. Kidney Int. 78, 1252-1262 (2010).

22. Ling, X. B. et al. Integrative urinary peptidomics in renal transplantation identifies biomarkers for acute rejection. J. Am. Soc. Nephrol. 21, 646-653 (2010).

23. Alkhalaf, A. et al. Multicentric validation of proteomic biomarkers in urine specific for diabetic nephropathy. PLOS ONE. 5, e13421 (2010).

24. Klein, J. et al. Urinary peptidomics provides a noninvasive humanized readout of diabetic nephropathy in mice. Kidney Int. 90, 1045-1055 (2016).

25. Good, D. M. et al. Naturally occurring human urinary peptides for use in diagnosis of chronic kidney disease. Mol. Cell Proteom. 9, 2424-2437 (2010).
26. Di Cera, E. Serine proteases. IUBMB Life 61, 510-515 (2009).

27. Cha, Y. H., Yook, J. I., Kim, H. S. \& Kim, N. H. Catabolic metabolism during cancer EMT. Arch. Pharm. Res. 38, 313-320 (2015).

28. Lovisa, $\mathrm{S}$. et al. Epithelial-to-mesenchymal transition induces cell cycle arrest and parenchymal damage in renal fibrosis. Nat. Med. 21, 998-1009 (2015).

29. Kern, U., Wischnewski, V., Biniossek, M. L., Schilling, O. \& Reinheckel, T. Lysosomal protein turnover contributes to the acquisition of TGFbeta-1 induced invasive properties of mammary cancer cells. Mol. Cancer 14, 39 (2015).

30. Finley, D. Recognition and processing of ubiquitin-protein conjugates by the proteasome. Annu. Rev. Biochem. 78, 477-513 (2009).

31. Goldberg, A. L. Development of proteasome inhibitors as research tools and cancer drugs. J. Cell Biol. 199, 583-588 (2012).

32. Dasgupta, S. et al. Proteasome inhibitors alter levels of intracellular peptides in HEK293T and SH-SY5Y cells. PLOS ONE 9, e103604 (2014).

33. Banno, A. et al. Downregulation of 265 proteasome catalytic activity promotes epithelial-mesenchymal transition. Oncotarget 7, 21527-21541 (2016).

34. Fricker, L. D., Gelman, J. S., Castro, L. M., Gozzo, F. C. \& Ferro, E. S. Peptidomic analysis of HEK293T cells: effect of the proteasome inhibitor epoxomicin on intracellular peptides. J. Proteome Res. 11, 1981-1990 (2012)

35. Larance, M., Ahmad, Y., Kirkwood, K. J., Ly, T. \& Lamond, A. I. Global subcellular characterization of protein degradation using quantitative proteomics. Mol. Cell. Proteom. 12, 638-650 (2013).

36. Kim, W. et al. Systematic and quantitative assessment of the ubiquitinmodified proteome. Mol. Cell 44, 325-340 (2011).

37. Gelman, J. S. et al. Alterations of the intracellular peptidome in response to the proteasome inhibitor bortezomib. PLOS ONE 8, e53263 (2013).

38. Chutipongtanate, S., Watcharatanyatip, K., Homvises, T., Jaturongkakul, K. \& Thongboonkerd, V. Systematic comparisons of various spectrophotometric and colorimetric methods to measure concentrations of protein, peptide and amino acid: detectable limits, linear dynamic ranges, interferences, practicality and unit costs. Talanta 98, 123-129 (2012).

39. Rawlings, N. D., Barrett, A. J. \& Finn, R. Twenty years of the MEROPS database of proteolytic enzymes, their substrates and inhibitors. Nucleic Acids Res. 44 D343-D350 (2016)

40. Fortelny, N., Yang, S., Pavlidis, P., Lange, P. F. \& Overall, C. M. Proteome TopFIND 3.0 with TopFINDer and PathFINDer: database and analysis tools for the association of protein termini to pre- and post-translational events. Nucleic Acids Res. 43, D290-D297 (2015).

41. Song, $X$. et al. SProtP: a web server to recognize those short-lived proteins based on sequence-derived features in human cells. PLOS ONE 6, e27836 (2011).

42. Szklarczyk, D. et al. STRINGv10: protein-protein interaction networks, integrated over the tree of life. Nucleic Acids Res. 43, D447-D452 (2015).

43. Valera, E., Dargusch, R., Maher, P. A. \& Schubert, D. Modulation of 5lipoxygenase in proteotoxicity and Alzheimer's disease. J. Neurosci. 33, 10512-10525 (2013) 